Research, Society and Development, v. 11, n. 12, e15111225392, 2022

(CC BY 4.0) | ISSN 2525-3409 | DOI: http://dx.doi.org/10.33448/rsd-v11i2.25392

\title{
Óleos essenciais de espécies Lamiaceae com potencial atividade Anti-fúngica: uma revisão
}

Essential Oills from Lamiaceae Species with potential Antifungal activity: a review

Aceites esenciales de especies de Lamiaceae con posible actividad Antifúngica: una revisión

Recebido: 05/01/2022 | Revisado: 09/01/2022 | Aceito: 19/01/2022 | Publicado: 21/01/2022

Denise Bezerra Correia

ORCID: https://orcid.org/0000-0001-6752-8705

Universidade Regional do Cariri, Brasil

E-mail: denisebezerra40@gmail.com

Dennis Bezerra Correia

ORCID: https://orcid.org/0000-0002-7782-4767

Universidade Regional do Cariri, Brasil

E-mail: denniscorreia40@gmail.com

Cícero Jorge Verçosa

ORCID: https://orcid.org/0000-0002-3284-6719

Secretaria de Educação e Esportes de Pernambuco, Brasil

E-mail: cjvercosa@hotmail.com

Maria Eliana Vieira Figueroa

ORCID: https://orcid.org/0000-0002-0049-4456

Secretaria de Educação e Esportes de Pernambuco, Brasil

E-mail: elianavfigueroa1@gmail.com

João Paulo Camilo de Oliveira

ORCID: https://orcid.org/0000-0003-0286-1149 Universidade Regional do Cariri, Brasi

E-mail: camilodeoliveirajoaopaulo35@gmail.com

Allyson Francisco dos Santos

ORCID: https://orcid.org/0000-0001-8635-6398

Secretaria de Educação e Esportes de Pernambuco, Brasil

E-mail: allysons@outlook.com.br

Nathália de Sousa Fernandes

ORCID: https://orcid.org/0000-0003-1079-5460

Universidade Regional do Cariri, Brasil

E-mail: natymusyc@gmail.com

Luiz Neldecílio Alves Vitor

ORCID: https://orcid.org/0000-0003-4085-4214

Universidade Federal do Ceará Brasil

E-mail: professorluizneldecilio@gmail.com Francisco Diego Pereira

ORCID: https://orcid.org/0000-0002-4816-3381

Universidade Regional do Cariri, Brasil

E-mail: diego.vilar.pereira@gmail.com

João Eudes Lemos de Barros

ORCID: https://orcid.org/0000-0003-1829-795X Universidade Regional do Cariri, Brasil

E-mail: joao.eudeslemos@urca.br Elizângela Beneval Bento

ORCID: https://orcid.org/0000-0002-7516-9711 Universidade Regional do Cariri, Brasil E-mail: elizangelaeliz@yahoo.com.br

Mayara Gonçalves Silva

ORCID: https://orcid.org/0000-0002-4568-2469

Centro Universitário Dr. Leão Sampaio, Brasil E-mail: maygoncalvesa@gmail.com Ginna Gonçalves Pereira

ORCID: https://orcid.org/0000-0003-0267-2615 Universidade Regional do Cariri, Brasil E-mail: ginna.pereira@urca.br

Cícero Leonardo Barbosa de Lima

ORCID: https://orcid.org/0000-0001-7798-0412

Universidade Regional do Cariri, Brasil

E-mail: leonardolimalima18@gmail.com

\section{Resumo}

Nos últimos anos, a incidência de infecções fúngicas tem aumentado intensamente. As leveduras de Candida são responsáveis por uma ampla variedade de manifestações clínicas, desde infecções do trato urinário até infecções da corrente sanguínea. Uma alternativa aos medicamentos estão os óleos essenciais e seus compostos bioativos, os quais 
podem apresentar amplo espectro antimicrobiano. Existe uma grande quantidade de trabalhos na literatura que abordam a atuação de óleos essenciais de espécies vegetais como fontes potenciais de agentes antimicrobianos, incluindo a ação anti-Candida. As espécies de Lamiaceae são ricas em óleos essenciais, os quais apresentam substâncias promissores na inibição de Candida. Esta revisão levantou os artigos publicados nos últimos cinco anos (2016 a 2021) com as principais atividades anti-Candida dos óleos essências dos representantes de Lamiaceae, além de ressaltar seus componentes majoritários. A revisão constou de dados obtidos de 56 artigos os quais incluía 71 plantas de Lamiaceae. Os óleos essenciais das espécies mostraram boa atividade anti-Candida (CIMs $<1000 \mu \mathrm{g} / \mathrm{mL}$ ). Os principais gêneros trabalhados foram: Thymus (14 artigos), Lavandula (7), Origanum (5) e Salvia (5). Os componentes majoritários encontrados nos óleos incluem timol (23 trabalhos), carvacrol (20), p-cimeno (17), linalol (10), 1,8-cineol (8), $\gamma$-terpineno (8) e $\alpha$-pineno (8) como principais substâncias. Os óleos essenciais podem representar um importante achado no controle de fungos multirresistentes a medicamentos.

Palavras-chave: Fungos; Thymus; Lavandula; Origanum; Concentração inibitória mínima (MIC).

\begin{abstract}
In recent years, the incidence of fungal infections has increased sharply. Candida yeasts are responsible for a wide variety of clinical manifestations, from urinary tract infections to bloodstream infections. An alternative to medicines are essential oils and their bioactive compounds, which can present a broad antimicrobial spectrum. There is a large amount of works in the literature that address the role of essential oils from plant species as potential sources of antimicrobial agents, including the anti-Candida action. The species of Lamiaceae are rich in essential oils, which show promising substances in the inhibition of Candida. This review raised articles published in the last five years (2016 to 2021) with the main anti-Candida activities of essential oils from representatives of Lamiaceae, in addition to highlighting their major components. The review consisted of data obtained from 56 articles which included 71 Lamiaceae plants. The essential oils of the species showed good anti-Candida activity (MICs $<1000 \mu \mathrm{g} / \mathrm{mL}$ ). The main genres worked were: Thymus (14 articles), Lavandula (7), Origanum (5) and Salvia (5). The major components found in the oils include thymol (23 works), carvacrol (20), p-cymene (17), linalool (10), 1,8-cineole (8), $\gamma$-terpinene (8) and $\alpha$-pinene ( 8) as main substances. Essential oils may represent an important finding in the control of multidrug-resistant fungi.
\end{abstract}

Keywords: Fungi; Thymus; Lavandula; Origanum; Minimum inhibitory concentration (MIC).

\title{
Resumen
}

In recent years, the incidence of fungal infections has increased sharply. Candida yeasts are responsible for a wide variety of clinical manifestations, from urinary tract infections to bloodstream infections. An alternative to medicines are essential oils and their bioactive compounds, which can present a broad antimicrobial spectrum. There is a large amount of works in the literature that address the role of essential oils from plant species as potential sources of antimicrobial agents, including the anti-Candida action. The species of Lamiaceae are rich in essential oils, which show promising substances in the inhibition of Candida. This review raised articles published in the last five years (2016 to 2021) with the main anti-Candida activities of essential oils from representatives of Lamiaceae, in addition to highlighting their major components. The review consisted of data obtained from 56 articles which included 71 Lamiaceae plants. The essential oils of the species showed good anti-Candida activity (MICs $<1000 \mu \mathrm{g} / \mathrm{mL}$ ). The main genres worked were: Thymus (14 articles), Lavandula (7), Origanum (5) and Salvia (5). The major components found in the oils include thymol (23 works), carvacrol (20), p-cymene (17), linalool (10), 1,8-cineole (8), $\gamma$-terpinene (8) and $\alpha$-pinene ( 8) as main substances. Essential oils may represent an important finding in the control of multidrug-resistant fungi.

Palabras clave: Fungi; Thymus; Lavandula; Origanum; Minimum inhibitory concentration (MIC).

\section{Introdução}

O número de infecções fúngicas tem aumentado nos últimos anos, principalmente em decorrência do aumento de pacientes de alto risco, que em sua maioria são hospedeiros debilitados ou imunocomprometidos. Tais infecções representam uma grave ameaça a assistência médica mundial, tendo as espécies de Candida como as principais causas de infecções fúngicas, resultando em uma taxa de mortalidade de 40\% a cada ano, em todo o mundo (Mccarty; Pappas, 2016; Tsay et al., 2020).

Candida albicans é o patógeno mais comumente relacionado a infecções fúngicas em humanos, responsável por cerca de metade dessas infecções, seguida por Candida tropicalis $(27,3 \%)$, Candida parapsilosis $(21,9 \%)$ e Candida glabrata $(4,4 \%)$ (LIU et al., 2014). Quando a função imunológica de um paciente está baixa, essas espécies podem causar infecções invasivas e se espalhar para órgãos internos. O desenvolvimento contínuo de novos tratamentos para as doenças, tem levado a um aumento no número de pacientes imunossuprimidos, bem como, cirurgias, internações longas em UTIs (unidades de terapia intensiva) e 
uso prévio de antibióticos de amplo espectro, os quais elevam o risco de candidíase disseminada (Spampinato; Leonardi, 2013; Medici; Poeta, 2015; Nami et al., 2019).

Apesar das drogas antifúngicas estarem disponíveis para infecções causadas por Candida, as taxas de mortalidade continuam crescendo. A utilização de novas classes de medicamentos antifúngicos não tem mostrado melhoras significativas no prognóstico de pacientes com infecções (Armstrong-James et al., 2017). Diversas classes de medicamentos têm aumentado progressivamente a resistência à Candida, em especial devido ao uso extensivo e prolongado de antibióticos, limitando consequentemente o número de agentes disponíveis (Pahwa et al., 2014; Marak; Dhanashree, 2018). Portanto, a ocorrência de resistência e a limitação do número de antifúngicos disponíveis, revelam a necessidade urgente de novos compostos ativos a fim de superar a resistência emergente aos fármacos (Dorsaz et al., 2017).

A busca por novos medicamentos com base em usos tradicionais e a identificação de novas moléculas com potencial antifúngico que sejam mais eficazes e menos tóxicas, é necessária para enfrentar o desafio da resistência aos fármacos antifúngicos (Dorsaz et al., 2017). Nesse contexto, as plantas medicinais, amplamente utilizadas por comunidades para o tratamento de diversas enfermidades se mostram promissoras no fornecimento de compostos ativos em medicamentos. As plantas e seus compostos, têm se mostrado eficazes no tratamento de diversas infecções, destacando-se as espécies da família Lamiaceae, ricas em óleos essências os quais apresentam diversas atividades biológicas, incluindo antimicrobiana, antifúngica, antioxidante, analgésica e anti-inflamatória (Najibzadeh; Yadegary, 2018; Salehi et al., 2018; Sharma et al., 2020).

Os vegetais possuem uma alta diversidade de substâncias bioativas de grande importância na indústria farmacêutica, a exemplo de antifúngicos como polienos e equinocandinas, os quais derivam diretamente de produtos naturais (Dorsaz et al., 2017). Considerando a importância dos compostos biossintetizados pelo metabolismo secundário dos vegetais, dentre os quais estão os óleos essenciais, sendo a família Lamiaceae ricas desses compostos, com essa revisão, objetiva-se levantar os artigos publicados nos últimos cinco anos (2016 a 2021) com as principais atividades anti-Candida dos óleos essências dos representantes desta família, além de ressaltar seus componentes majoritários.

\section{Metodologia}

A palavra-chave "Lamiaceae" foi associada aos termos "atividade antifúngica", "atividade anti-Candida", "Candida" e “essential oil" para a busca de publicações de 2016 a 2021. Para tanto, foram utilizadas as plataformas Scopus e Web of Science. Os nomes das espécies foram verificados e confirmados no The Plant List. Após a busca, foi feita a leitura dos resumos e seleção dos artigos. Os nomes dos compostos químicos foram unificados, de acordo com PubChem. A Figura 1 resume o processo de busca e os resultados obtidos. 
Figura 1. Fluxograma do processo de busca e seleção dos artigos.

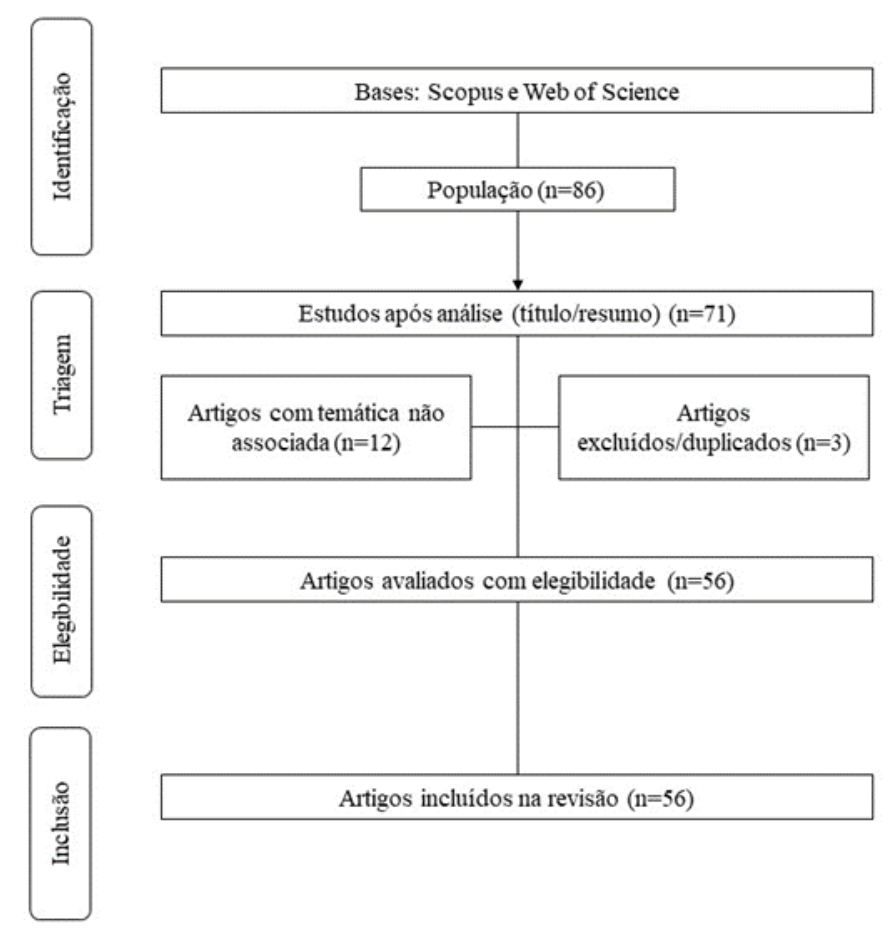

Fonte: Autores (2021).

\section{Resultados e Discussão}

No total, 56 artigos (de 2016 a 2021) foram incluídos na revisão. A tabela 1 apresenta as espécies vegetais (71) testadas contra Candida. A maioria dos estudos foram conduzidos com o gênero Thymus (14 artigos), seguido por Lavandula (7) e Origanum e Salvia, com 5 trabalhos cada. Foi demonstrado em trabalho anterior que os óleos essências das espécies de tais gêneros estão entre os mais frequentemente investigados (Potente et al., 2020).

Representada por cerca de 236 gêneros e mais de 7500 espécies, Lamiaceae está entre uma das principais famílias de plantas que produzem óleos essenciais. Tais compostos, são sintetizados naturalmente por espécies aromáticas e conhecidos por estarem envolvidos na proteção do vegetal, atuando contra herbívoros, pragas, bactérias e fungos (Galvan et al., 2021). É notável a complexidade química dos óleos essenciais, sendo que pouco mais de 300 substâncias distintas podem estar presentes em um óleo essencial. Destes, os compostos terpênicos são predominantes, entre eles, os mono e sesquiterpenos são os mais abundantes. Além disso, os óleos essenciais obtidos das espécies de Lamiaceae são largamente utilizados como terapias alternativas devido os seus efeitos antimicrobianos. A ampla gama de propriedades medicinais dos óleos essenciais torna-os fortes candidatos para as indústrias farmacêuticas na busca de compostos antimicrobianos ativos (Iseppi et al., 2020).

Os compostos químicos encontrados nos óleos essências das espécies de Lamiaceae apresentadas na Tabela 1, incluem timol (23 trabalhos), carvacrol (20), p-cimeno (17), linalol (10), 1,8-cineol (8), $\gamma$-terpineno (8) e $\alpha$-pineno (8) como principais compostos (Figura 2). Segundo Bona et al. (2016) óleos essenciais com altas concentrações de monoterpenos (exemplo: timol, carvacrol e p-cimeno) possuem importantes atividades antifúngicas. Origanum e Thymus estão entre os gêneros mais ricos nesses compostos, os quais foram os principais representantes vegetais testados contra Candida nos artigos analisados neste trabalho. Compostos como linalol e 1,8-cineol cíclico, terpinenos e pinenos também são outros monoterpenos frequentemente identificados em Lamiaceae, associados também, a atividades anti-Candida (Karpiński, 2020). Devido à complexidade química 
dos óleos essências e dado que os efeitos biológicos são frequentemente advindos de uma interação sinérgica que ocorre entre os diversos constituintes, é difícil identificar exclusivamente as substâncias mais ativas (Potente et al., 2020).

Figura 2. Fórmulas químicas das sete substâncias mais comumente encontradas em óleos essenciais de espécies de Lamiaceae.<smiles>Cc1ccc(C(C)C)c(O)c1</smiles>

Timol<smiles>Cc1ccc(C(C)C)cc1O</smiles>

Carvacrol<smiles>Cc1ccc(C(C)C)cc1</smiles>

p-cimeno<smiles>CC12CCC(CC1)C(C)(C)O2</smiles>

1,8-cineol<smiles>C=C[C@@](C)(O)CCC=C(C)C</smiles>

Linalol<smiles>CC1=CCC(C(C)C)=CC1</smiles>

$\gamma$-terpineno<smiles>CC1=CCC2CC1C2(C)C</smiles>

$\alpha$-pineno

Fonte: Autores.

Quanto as cepas analisadas nos estudos das atividades anti-Candida dos óleos das espécies de Lamiaceae (Tabela 1), pode-se observar que C. albicans (65 trabalhos) foi a espécie utilizada em todos os artigos verificados. Candida albicans é a espécie mais prevalente entre Candida spp., sendo considera a principal levedura patogênica oportunista, responsável por infecções superficiais e sistêmicas, a espécie é considerada um organismo modelo nos estudos de patógenos fúngicos (Marak; Dhanashree, 2018). Demais cepas como C. tropicalis (12) e Candida parapsilosis (7) também foram as cepas mais utilizadas nos ensaios dos artigos analisados. Candida tropicalis foi considerada por outros autores como a segunda espécie de Candida mais virulenta, seguida apenas por $C$. albicans, sendo ainda, reconhecida como grande produtor de biofilme (Zuza-Alves; Silva-Rocha; Chaves, 2017). Candida parapsilosis também é o segundo ou terceiro agente etiológico de Candida mais comumente isolado em unidades de terapia intensiva (UTI) (Guo et al., 2021).

Na Tabela 1 são mostrados os valores de MIC (Concentração Inibitória Mínima) dos óleos essenciais de Lamiaceae em cepas de Candida. A maioria dos óleos apresentam boa atividade $(<1000 \mu \mathrm{g} / \mathrm{mL})$ contra os fungos testados. Em alguns casos foram verificadas discrepâncias entre os diferentes estudos. Por exemplo, a ação dos óleos essenciais de Lavandula dentata contra Candida albicans, onde no trabalho de Justus et al (2018) a MIC atingiu $54.7 \mu \mathrm{g} / \mathrm{mL}$, enquanto em Müller-Sepúlveda et al. (2020) as MICs estavam entre 156 a 130 $\mu \mathrm{g} / \mathrm{mL}$. As diferenças podem estar relacionadas ao teor dos componentes e às diferentes composições químicas dos óleos essenciais avaliados (Karpiński, 2020). Nos resultados apresentados por Justus et al (2018) os principais compostos dos óleos essenciais foi 1,8-cineol (63\%), enquanto em Müller-Sepúlveda et al. (2020) as duas substâncias principais foram eucaliptol (60.85\%) e $\beta$-Pineno (9.89\%). Os diferentes valores de MIC observados dos óleos das espécies de Lamiaceae possa se dever, dentre outros fatores, às distintas regiões de coleta do espécime avaliado, à composição química e a metodologia de pesquisa utilizada (Karpiński, 2020). 
Tabela 1. Espécies de Lamiaceae produtoras de óleos essências com atividade anti-Candida.

\begin{tabular}{|c|c|c|c|}
\hline Espécie vegetal & Constituintes majoritários & Candida / MIC & Referência \\
\hline Aeollanthus heliotropioides & $\begin{array}{c}\text { linalol } \\
(38,5 \%), \text { Z-a-farneseno } \\
(25,1 \%), \text { 9-hexa-decen-1-ol } \\
(13,9 \%)\end{array}$ & 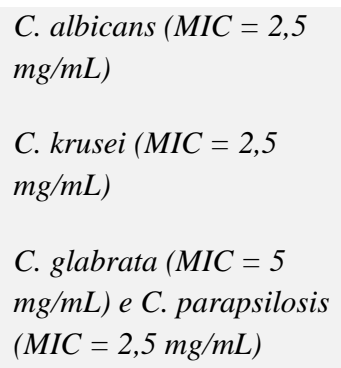 & Mback et al., 2016 \\
\hline Ajuga turkestanica & $\begin{array}{c}\beta \text {-Linalool }(26,6 \%), \alpha \text {-terpineol } \\
(10,0 \%) \text {, cumarina }(8,9 \%) \\
\text { e } 4,5,7,7 \alpha \text {-tetrahidro- } 4,4,7 \alpha \text { - } \\
\text { trimetil-2 }(6 \mathrm{H}) \text {-benzofuranona } \\
(5,4 \%)\end{array}$ & C. albicans & Mamadalieva et al., 2019 \\
\hline Calamintha glandulosa & $\begin{array}{l}\text { Mentona ( } 15.7 \%) \text {, Pulegone } \\
(35.1 \%) \text { e piperitona }(23.4 \%)\end{array}$ & $\begin{array}{l}\text { C. albicans } \\
(M I C=>186 \mu \mathrm{g} / \mathrm{mL})\end{array}$ & Milenković et al., 2018 \\
\hline Calamintha nepeta & $\begin{array}{c}\text { pulegone }(58 \%) \text { e piperitenona } \\
\qquad(27.4 \%)\end{array}$ & $\begin{array}{l}\text { C. albicans } \\
(M I C=>191 \mu \mathrm{g} / \mathrm{mL})\end{array}$ & Milenković et al., 2018 \\
\hline Calamintha sylvatica & $\begin{array}{c}\text { epóxido cis-piperitona (63.3\%) } \\
\text { e mentona }(10.8 \%)\end{array}$ & $\begin{array}{l}\text { C. albicans } \\
(M I C=>182 \mu \mathrm{g} / \mathrm{mL})\end{array}$ & Milenković et al., 2018 \\
\hline Calamintha vardarensis & $\begin{array}{c}\text { Pulegone (51.6\%) e mentona } \\
(19.9)\end{array}$ & $\begin{array}{l}\text { C. albicans } \\
(M I C=>170 \mu \mathrm{g} / \mathrm{mL})\end{array}$ & Milenković et al., 2018 \\
\hline Clinopodium nepeta & $\begin{array}{c}\text { óxido de piperitona }(51,7 \%) \text { e } \\
\text { óxido }(23,4 \%)\end{array}$ & $\begin{array}{l}\text { C. albicans }(M I C=0.4 \\
m g / m L)\end{array}$ & Debbabi et al., 2020 \\
\hline Coridothymus capitatos & timol $(65,6 \%)$ & $\begin{array}{l}\text { C. albicans }(M I C=0,8 \\
m g / m L)\end{array}$ & Hafi et al., 2017 \\
\hline Coridothymus capitatus & $\begin{array}{c}\text { Timol }(29,3 \%) \text { e Carvacrol } \\
(29,3 \%)\end{array}$ & $\begin{array}{l}\text { C. albicans } \\
(M I C=128 \mu \mathrm{g} / \mathrm{mL})\end{array}$ & Khoury et al., 2016 \\
\hline Coridothymus capitatus & $\begin{array}{l}\text { carvacrol }(67,58 \%) \text { e timol } \\
\qquad(0,16 \%) \\
\text { seguido por monoterpenos } \\
\text { alcoólicos, como } \beta \text {-linalol } \\
(0,97 \%), \text { L-terpinen-4-ol } \\
(0,93 \%), \text { borneol }(0,48 \%) \text { e } \alpha \text { - } \\
\text { terpineol }(0,09 \%)\end{array}$ & $\begin{array}{l}\text { C. albicans }(M F C= \\
0,125 \mu \mathrm{g} / \mathrm{mL}), C . \text { krusei } \\
(M F C=0,250 \mu \mathrm{g} / \mathrm{mL}), \\
\text { C. glabrata }(M F C= \\
0,125 \mu \mathrm{g} / \mathrm{mL}), \\
\text { C. norvegensis }(M F C= \\
0,250 \mu \mathrm{g} / \mathrm{mL}), \\
\text { C. lusitaniae }(M F C= \\
0,250 \mu \mathrm{g} / \mathrm{mL}),\end{array}$ & Marino et al., 2020 \\
\hline
\end{tabular}




\begin{tabular}{|c|c|c|c|}
\hline & & $\begin{array}{l}\text { C. valida }(M F C=0,250 \\
\mu g / m L), \\
\text { C. } \text { guillermondii }(M F C= \\
0,250 \mu \mathrm{g} / \mathrm{mL}) \text {, } \\
\text { C. parapsilosis }(M F C= \\
0,125 \mu \mathrm{g} / \mathrm{mL}) . \\
\text { C. tropicalis }(M F C= \\
0,125 \mu \mathrm{g} / \mathrm{mL}) .\end{array}$ & \\
\hline $\begin{array}{l}\text { Dracocephalum } \\
\text { integrifolium }\end{array}$ & $\begin{array}{c}\text { sabinene } \\
(7,35-14,0 \%) \text { e eucaliptol } \\
(53,56-76,11 \%)\end{array}$ & $\begin{array}{l}\text { C. albicans }(M I C= \\
4,347-37,712 \mathrm{mg} / \mathrm{mL})\end{array}$ & Zhou et al., 2019 \\
\hline Dracocephalum kotschyi & $\begin{array}{c}\alpha \text {-pineno. } \\
(13,66 \%),(\mathrm{E})-\operatorname{citral}(12,89 \%), \\
\text { neral }(11,25 \%)\end{array}$ & $\begin{array}{l}\text { C. albicans }(M I C=31,25 \\
\mu g / m l)\end{array}$ & Ghavam et al., 2021 \\
\hline Hyssopus officinalis & $\begin{array}{c}\text { pinocamfona }(50,0 \pm 2 \%) \text {, } \\
\text { izopinocanfeno }(28,0 \pm 1 \%) \text { e } \\
\alpha \text {-pineno }(11,0 \pm 1 \%)\end{array}$ & C. albicans & Proškovcová et al., 2021 \\
\hline Lavandula angustifolia & linalol $(45,8 \%)$ & $\begin{array}{l}\text { C. albicans } \\
(M I C=512 \mu \mathrm{g} / \mathrm{mL})\end{array}$ & Khoury et al., 2016 \\
\hline Lavandula angustifolia & - & C. albicans & Andrys et al., 2018 \\
\hline Lavandula dentata & 1,8-cineol $(63 \%)$ & $\begin{array}{l}\text { C. albicans } \\
(M I C=54.7 \mu \mathrm{g} / \mathrm{mL})\end{array}$ & Justus et al., 2018 \\
\hline Lavandula dentata & $\begin{array}{c}\text { eucaliptol (60.85\%) e } \beta \text {-Pineno } \\
\qquad \begin{array}{c}( \\
9.89 \\
\%)\end{array}\end{array}$ & $\begin{array}{l}\text { C. albicans } \\
(M I C=156 e \\
130 \mu \mathrm{g} / \mathrm{mL})\end{array}$ & Müller-Sepúlveda et al., 2020 \\
\hline Lavandula stoechas & $\alpha$-fenchone $(26,2 \%)$ & $\begin{array}{l}\text { C. albicans } \\
(M I C=512 \mu \mathrm{g} / \mathrm{mL})\end{array}$ & Khoury et al., 2016 \\
\hline $\begin{array}{l}\text { Lavandula stoechas subsp. } \\
\text { luisieri }\end{array}$ & $\begin{array}{l}\text { Monoterpenos oxigenados, } \\
\text { tais como 1,8-cineol, } \\
\text { lavandulol e derivados de } \\
\text { necrodano }\end{array}$ & $\begin{array}{l}\text { C. albicans } \\
\text { C. krusei } \\
\text { C. parapsilosis }\end{array}$ & Arantes et al., 2016 \\
\hline Lavandula $x$ intermedia & $\begin{array}{c}\text { linalol }(36,0 \%), \text { acetato de } \\
\text { linalila }(27,3 \%)\end{array}$ & $\begin{array}{l}\text { C. albicans } \\
(M I C=18 \text { to } 144 \mu \mathrm{g} / \mathrm{mL})\end{array}$ & Iseppi et al., 2020 \\
\hline Lepechinica mutica & $\begin{array}{c}\text { Hidrocarbonetos } \\
\text { sesquiterpênicos }(38,50 \%) \text { e }\end{array}$ & $\begin{array}{l}\text { C. albicans }(M I C>9 \\
m g / m l)\end{array}$ & Ramirez et al., 2017 \\
\hline
\end{tabular}


Research, Society and Development, v. 11, n. 12, e15111225392, 2022

(CC BY 4.0) | ISSN 2525-3409 | DOI: http://dx.doi.org/10.33448/rsd-v11i2.25392

\begin{tabular}{|c|c|c|c|}
\hline & $\begin{array}{c}\text { hidrocarbonetos } \\
\text { monoterpênicos }(30,59 \%)\end{array}$ & & \\
\hline $\begin{array}{l}\text { Melissa officinalis subsp. } \\
\text { Altissima }\end{array}$ & $\begin{array}{c}\text { óxido de cariofileno }(38,9 \%) \text { e } \\
\text { pulegone }(70,5 \%)\end{array}$ & $\begin{array}{l}\text { C. albicans (MIC - 6,24 a } \\
12,48 \mathrm{mg} / \mathrm{mL})\end{array}$ & Božović et al., 2018 \\
\hline (Sibth. \& Sm.) Arcang. & & & \\
\hline Mentha arvensis & $\begin{array}{l}\text { mentol }(73,8 \%), \text { mentona } \\
(7,8 \%), \text { isomentona }(5,4 \%)\end{array}$ & $\begin{array}{l}\text { C. albicans } \\
(M I C=18 \text { to } 144 \mu \mathrm{g} / \mathrm{mL})\end{array}$ & Iseppi et al., 2020 \\
\hline Mentha pulegium & $\begin{array}{c}\text { pulegona }(50,6 \%) \text {, piperitenona } \\
(27,8 \%) \text { e mentona }(6,9 \%)\end{array}$ & $\begin{array}{l}\text { C. albicans (MIC - } \\
6.25 \mu \mathrm{g} / \mathrm{mL})\end{array}$ & Casiglia et al., 2017 \\
\hline Mentha spicata & Pulegone $(78,7 \%)$ & $\begin{array}{l}\text { C. albicans } \\
(M I C=>512 \mu \mathrm{g} / \mathrm{mL})\end{array}$ & Khoury et al., 2016 \\
\hline Mesosphaerum suaveolens & $\begin{array}{c}\beta \text {-Caryophyllene }(18.6 \%), \\
\text { Sabinene }(15.92 \%) \\
\text { spathulenol }(11.08 \%)\end{array}$ & $\begin{array}{l}\text { C.albicans (IC50 - 18,15 } \\
\mu \mathrm{g} / \mathrm{mL}) \\
\text { C.tropicalis (IC50 - 40,4 } \\
\mu \mathrm{g} / \mathrm{mL})\end{array}$ & Bezerra et al., 2020 \\
\hline $\begin{array}{l}\text { Micromeria fruticosa } \\
\text { serpyllifolia }\end{array}$ & pulegonas e isomentonas & $\begin{array}{l}\text { C. albicans }(M I C=0.206 \\
m g / m L)\end{array}$ & Salameh et al., 2020 \\
\hline Micromeria graeca & $\begin{array}{c}\text { geranial }(36,93 \%) \\
\text { z-citral }(18,25 \%), 1,8 \text {-epoxi- } \\
\text { pmenth-2-eno }(13,01 \%) \text {, }\end{array}$ & $\begin{array}{l}\text { C. albicans } \\
\text { (MIC - 3,12 mg / mL) }\end{array}$ & Benali et al., 2021 \\
\hline Monarda didyma & $\begin{array}{c}\text { timol }(62 \%) \text { e p-cimeno } \\
(10,22 \%)\end{array}$ & $\begin{array}{l}\text { C. albicans } \\
\text { C. tropicalis } \\
\text { C. pseudointermedia } \\
\text { C. stellata }\end{array}$ & Mattarelli et al., 2017 \\
\hline Monarda didyma & $\begin{array}{c}\text { Timol }(47,54 \%) \text { e eucaliptol } \\
\qquad(17,82 \%)\end{array}$ & C. albicans & Shanaida et al., 2021 \\
\hline Monarda fistulosa & $\begin{array}{c}\text { timol }(31 \%) \text { e } \beta \text {-felandreno } \\
(17 \%)\end{array}$ & $\begin{array}{l}\text { C. albicans } \\
\text { C. tropicalis } \\
\text { C. pseudointermedia } \\
\text { C. stellata }\end{array}$ & Mattarelli et al., 2017 \\
\hline Nepeta sintenisii & $\begin{array}{c}\text { 4a,7,7a-Nepetalactone } \\
\qquad(51.74 \%), \\
\text {-Farnesene }(12.26 \%), 4 \mathrm{a}, 7,7 \mathrm{a}- \\
\text { Nepetalactone }(8.01 \%), \\
\text { Germacrene-D }(5.01 \%)\end{array}$ & $\begin{array}{l}\text { C. albicans }(M I C<0.35 \\
\mu g / m L)\end{array}$ & Shakeri et al., 2016 \\
\hline $\begin{array}{l}\text { Ocimum } \\
\text { tenuiflorum }\end{array}$ & $\begin{array}{l}\text { metil eugenol }(84,7 \%) \text { e } \beta \text { - } \\
\quad \text { cariofileno }(7,4 \%)\end{array}$ & $\begin{array}{l}\text { C. albicans }(M I C=1.25 \\
\mu L / m L),\end{array}$ & \\
\hline
\end{tabular}




\begin{tabular}{|c|c|c|c|}
\hline & & $\begin{array}{l}\text { C. tropicalis }(M I C=2.5- \\
1.25 \mu L / m L) \text {, } \\
\text { C. } \text { krusei }(M I C=1.25 \\
\mu L / m L) \text {, } \\
\text { C. guillermondii }(M I C= \\
\text { 1.25 } \mu \mathrm{L} / \mathrm{mL}) \text { e } \\
\text { C. parapsilosis }(M I C= \\
1.25 \mu L / m L)\end{array}$ & \\
\hline Ocimum basilicum & linalol $(46,0 \%)$ & C. albicans e C. famata & Ebani et al., 2018 \\
\hline Ocimum basilicum & $\begin{array}{c}\text { Linalol }(35,1 \%) \text {, eugenol } \\
(20,7 \%) \text { e } 1,8 \text {-cineol }(9,9 \%)\end{array}$ & $\begin{array}{l}\text { C. albicans }(M I C=0.64 \\
\mu L / m L) \text {, } \\
\text { C. tropicalis }(M I C=0.64 \\
\mu L / m L) \text {, } \\
\text { C. } \text { krusei }(M I C=0.64 \\
\mu L / m L) \text {, } \\
\text { C. guillermondii }(M I C= \\
\text { O.64 } \mu \mathrm{L} / \mathrm{mL}) \text { e } \\
\text { C. parapsilosis }(M I C= \\
0.64 \mu \mathrm{L} / \mathrm{mL})\end{array}$ & 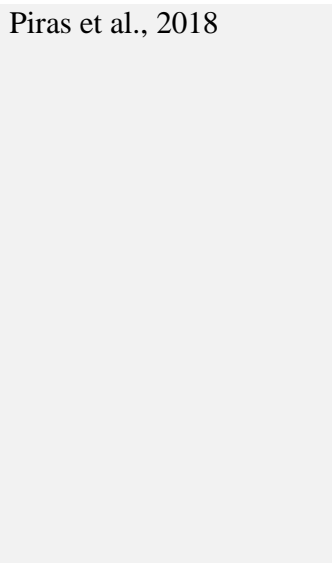 \\
\hline Ocimum basilicum & $\begin{array}{c}\text { linalol }(31,6 \%) \text { e metil chavicol } \\
\qquad(23,8 \%)\end{array}$ & C. albicans & Stanojevic et al., 2017 \\
\hline Origanum minutiflorum & carvacrol $(90,87 \%)$ & C. albicans & Albayrak; Aksoy, 2019 \\
\hline Origanum syriacum & Timol $(74,4 \%)$ & $\begin{array}{l}\text { C. albicans } \\
(M I C=128 \mu \mathrm{g} / \mathrm{mL})\end{array}$ & Khoury et al., 2016 \\
\hline Origanum vulgare & $\begin{array}{c}\text { carvacrol }(57,3 \%), 1,8 \text {-cineol } \\
\qquad(12,9 \%) \mathrm{e} \\
\alpha \text {-pineno }(7,9 \%)\end{array}$ & $\begin{array}{l}\text { C. albicans } \\
(M I C=250-500 \mathrm{mg} / \mathrm{L}) \\
\text { C. glabrata }(M I C=500- \\
1000 \mathrm{mg} / \mathrm{L})\end{array}$ & Baj et al., 2020 \\
\hline Origanum vulgare & $\begin{array}{l}\text { carvacrol } \\
(65,9 \%)\end{array}$ & C. albicans e C. famata & Ebani et al., 2018 \\
\hline Origanum vulgare & $\begin{array}{c}\text { terpinen- } \\
\text { 4-ol }(38,35 \%) \text { e hidrato de } \\
\text { trans-sabineno }(10,06 \%)\end{array}$ & $\begin{array}{l}\text { C. albicans }(M I C=2.36 \\
m g / m L)\end{array}$ & Fikry et al., 2019 \\
\hline Origanum vulgare & carvacrol $(85,0 \pm 3 \%)$ & C. albicans & Proškovcová et al., 2021 \\
\hline Phlomis fruticosa & - & $\begin{array}{l}\text { C. albicans e } C \text {. } \\
\text { tropicalis }\end{array}$ & Ferrante et al., 2019 \\
\hline Phlomis herba-venti & - & C. albicans e $C$. & Ferrante et al., 2019 \\
\hline
\end{tabular}




\begin{tabular}{|c|c|c|c|}
\hline & & tropicalis & \\
\hline Phlomis kurdica & $\begin{array}{c}\text { germacreno D }(55,4 \%),(Z)-\beta- \\
\text { farneseno }(11,2 \%), \\
\text { e ácido hexadecanóico }(8,4 \%)\end{array}$ & $\begin{array}{l}\text { C. albicans } \\
(M I C=>1000 \mu \mathrm{g} / \mathrm{mL})\end{array}$ & Karadağ et al., 2020 \\
\hline Phlomis regelii & $\begin{array}{c}\text { canfeno }(17,1 \%), 1,8 \text {-cineol } \\
(15,9 \%), \beta \text {-cimeno }(7,9 \%)\end{array}$ & C. albicans & Mamadalieva et al., 2019 \\
\hline $\begin{array}{l}\text { Plectranthus } \\
\text { amboinicus }\end{array}$ & $\begin{array}{c}\text { carvacrol }(71 \%) \text { e Para-cimeno } \\
(9.7 \%)\end{array}$ & $\begin{array}{l}\text { C. albicans } \\
\left(I C_{50}=>64 \mu \mathrm{g} / \mathrm{mL}\right)\end{array}$ & Monzote et al., 2020 \\
\hline Rhaphiodon echinus & $\begin{array}{c}\text { biciclogermacreno }(28,13 \%) \text {, } \\
\beta \text {-cariofileno } \\
(23,07 \%) \text { óxido de cariofileno } \\
(5,40 \%)\end{array}$ & $\begin{array}{l}\text { C. albicans, C. krusei e } C \text {. } \\
\text { tropicalis }\end{array}$ & Duarte et al., 2016 \\
\hline Rosmarinus officinalis & Eucaliptol $(21,5 \%)$ & $\begin{array}{l}\text { C. albicans } \\
(M I C=512 \mu \mathrm{g} / \mathrm{mL})\end{array}$ & Khoury et al., 2016 \\
\hline Rosmarinus officinalis & $\begin{array}{c}\text { eucaliptol }(39,0 \%) \text {, cânfora } \\
(13,7 \%), \alpha \text {-pineno }(10,3 \%), \\
(1 S)-(-) \text { - } \beta \text {-pineno }(7,2 \%)\end{array}$ & $\begin{array}{l}\text { C. albicans (MIC - 1,2 - } 5 \\
m g / m L)\end{array}$ & Bogavac et al., 2017 \\
\hline Rosmarinus officinalis & 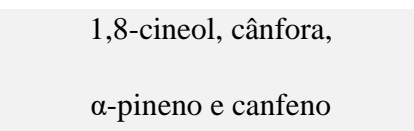 & C. albicans & Cutillas et al., 2018 \\
\hline Rosmarinus officinalis & - & $\begin{array}{l}\text { C. albicans }(M I C=600 \\
\mu g / m L)\end{array}$ & Lorenzo-Leal et al., 2019 \\
\hline Rosmarinus officinalis & $\begin{array}{l}\text { 1,8-cineol }(25,0 \pm 1 \%) \alpha- \\
\quad \text { pineno }(19,0 \pm 1 \%)\end{array}$ & C. albicans & Proškovcová et al., 2021 \\
\hline Salvia fruticosa & Eucaliptol $(57,3 \%)$ & $\begin{array}{l}\text { C. albicans } \\
(M I C=512 \mu \mathrm{g} / \mathrm{mL})\end{array}$ & Khoury et al., 2016 \\
\hline Salvia officinalis & $\begin{array}{c}\rho \text {-cimeno }(40,0 \pm 3 \%) \text { timol } \\
(32,0 \pm 2 \%)\end{array}$ & C. albicans & Proškovcová et al., 2021 \\
\hline Salvia palaestina & $\begin{array}{l}\text { eucaliptol }(47,09 \%) \text { e cânfora } \\
\qquad(8,73 \%)\end{array}$ & $\begin{array}{l}\text { C. albicans }(M I C= \\
14.04 \mathrm{mg} / \mathrm{mL})\end{array}$ & Sabbobeh et al., 2016 \\
\hline Salvia sclarea & acetato de linalila $(54,7 \%)$ & C. albicans e C. famata & Ebani et al., 2018 \\
\hline Salvia sclarea & $\begin{array}{c}\text { acetato de linalil } \\
(45,51 \%) \text { e linalol }(38,98 \%)\end{array}$ & C. albicans & Shanaida et al., 2021 \\
\hline Salvia staminea & $\begin{array}{c}\text { acetato de linalil }(23,30 \%) \mathrm{e} \\
\text { linalol }(22,05 \%)\end{array}$ & C. albicans & Çolak et al., 2018 \\
\hline Satureja bachtiarica & p-cimeno $(13.56 \%)$ & $\begin{array}{l}\text { C. albicans } \\
(M I C=0.06 \mu \mathrm{g} / \mathrm{mL})\end{array}$ & Jafari et al.,2018 \\
\hline
\end{tabular}


Research, Society and Development, v. 11, n. 12, e15111225392, 2022

(CC BY 4.0) | ISSN 2525-3409 | DOI: http://dx.doi.org/10.33448/rsd-v11i2.25392

\begin{tabular}{|c|c|c|c|}
\hline Satureja bachtiarica & $\begin{array}{l}\text { carvacrol }(54,95-65,48 \%), \\
\text { timol }(12-15,70 \%), \gamma \text {-terpineno } \\
(4,55-13,55 \%)\end{array}$ & C. albicans & Alizadeh, 2016 \\
\hline Satureja cuneifolia & carvacrol $(62,4 \%)$ & $\begin{array}{l}\text { C. albicans }(M I C=0,4 \\
m g / m L)\end{array}$ & Hafi et al., 2017 \\
\hline Satureja cuneifolia & Carvacrol $(69,5 \%)$ & $\begin{array}{l}\text { C. albicans } \\
(M I C=128 \mu \mathrm{g} / \mathrm{mL})\end{array}$ & Khoury et al., 2016 \\
\hline Satureja hortensis & p-cimeno $(13.07 \%)$ & $\begin{array}{l}\text { C. albicans } \\
(M I C=0.12 \mu \mathrm{g} / \mathrm{mL})\end{array}$ & Jafari et al.,2018 \\
\hline Satureja hortensis & $\begin{array}{l}\text { carvacrol }(50,7 \%), \gamma \text {-terpineno } \\
(24,3 \%) \text { e p-cimeno }(12,5 \%)\end{array}$ & $\begin{array}{l}\text { C. albicans } \\
(M I C=250-500 \mathrm{mg} / \mathrm{L}) \\
\begin{array}{l}\text { C. } \text { glabrata }(M I C=500 \\
\mathrm{mg} / \mathrm{L})\end{array}\end{array}$ & Baj et al., 2020 \\
\hline Satureja intermedia & p-cimeno $(17.50 \%)$ & $\begin{array}{l}\text { C. albicans } \\
(M I C=0.25 \mu \mathrm{g} / \mathrm{mL})\end{array}$ & Jafari et al.,2018 \\
\hline Satureja laxiflora & p-cimeno $(16,95 \%)$ & $\begin{array}{l}\text { C. albicans } \\
(M I C=0.25 \mu \mathrm{g} / \mathrm{mL})\end{array}$ & Jafari et al.,2018 \\
\hline Satureja thymbra & timol $(54,3 \%)$ & $\begin{array}{l}\text { C. albicans }(M I C=0,4 \\
m g / m L)\end{array}$ & \\
\hline Satureja thymbra & Timol $(44,5 \%)$ & C. albicans & Khoury et al., 2016 \\
\hline Siderite cipriota & $\begin{array}{l}\text { epi-cubebol }(11,9 \%) \text {, trans- } \\
\text { piperitol } \\
(8,9 \%) \text { e } \alpha \text { pineno }(8,1 \%)\end{array}$ & C. albicans & Hanoğlu et al.,2017 \\
\hline Sideritis cesarea & $\begin{array}{l}\text { óxido de cariofileno }(13,7 \%), \\
\text { ácido hexadecanóico } \\
(8,5 \%) \text { e espatulenol }(6,1 \%)\end{array}$ & $\begin{array}{l}\text { C. albicans } \\
(M I C=6 \mathrm{mg} / \mathrm{mL})\end{array}$ & Günbatan et al., 2017 \\
\hline $\begin{array}{l}\text { Sideritis romana } \\
\text { L. subsp. purpurea (Tal. ex } \\
\text { Benth.) }\end{array}$ & $\begin{array}{c}\text { biciclogermacreno }(23,8 \%) \\
\text { germacreno D }(8 \%),(\mathrm{E})- \\
\text { cariofileno }(7,9 \%) \mathrm{e} \\
\text { espatulenol }(5,5 \%)\end{array}$ & $\begin{array}{l}\text { C. albicans }(M I C=2,46 \\
m g / m L)\end{array}$ & Tadi'c et al., 2017 \\
\hline Sideritis trojana & $\begin{array}{c}\text { valeranona } \\
(11,3 \%), \alpha \text {-bisabolol }(10,9 \%) \mathrm{e} \\
\beta \text {-cariofileno }(8,8 \%)\end{array}$ & $\begin{array}{l}\text { C. albicans } \\
(M I C=>1000 \mu \mathrm{g} / \mathrm{mL})\end{array}$ & Kirmizibekmez et al., 2017 \\
\hline Stachys byzantina & $\begin{array}{l}\text { hexahidrofarnesil acetona } \\
\qquad(20,41 \%)\end{array}$ & $\begin{array}{l}\text { C. albicans } \\
(M I C=500 \mu \mathrm{g} / \mathrm{mL})\end{array}$ & Kiashi et al., 2020 \\
\hline Stachys laxa & Ácido hexadecanóico $(16,65 \%)$ & $\begin{array}{l}\text { C. albicans } \\
(M I C=250 \mu \mathrm{g} / \mathrm{mL})\end{array}$ & Kiashi et al., 2020 \\
\hline
\end{tabular}


Research, Society and Development, v. 11, n. 12, e15111225392, 2022

(CC BY 4.0) | ISSN 2525-3409 | DOI: http://dx.doi.org/10.33448/rsd-v11i2.25392

\begin{tabular}{|c|c|c|c|}
\hline Thymbra spicata & Carvacrol $(60,9 \%)$ & $\begin{array}{l}\text { C. albicans }(M I C=0,6 \\
m g / m L)\end{array}$ & Hafi et al., 2017 \\
\hline Thymbra spicata & Carvacrol $(64,0 \%)$ & $(M I C=128 \mu \mathrm{g} / \mathrm{mL})$ & Khoury et al., 2016 \\
\hline $\begin{array}{l}\text { Thymus } \\
\text { serpyllum }\end{array}$ & $\begin{array}{c}\text { linalol }(30,9 \%) \\
\text { e timol }(25,1 \%) \text {, geraniol } \\
(10,5 \%)\end{array}$ & $\begin{array}{l}\text { C. albicans } \\
(M I C=1000-2000 \\
\mathrm{mg} / \mathrm{L}) \\
\text { C. glabrata }(\text { MIC }=1000 \\
-4000 \mathrm{mg} / \mathrm{L})\end{array}$ & Baj et al., 2020 \\
\hline $\begin{array}{l}\text { Thymus } \\
\text { seravschanicus }\end{array}$ & $\begin{array}{c}\text { timol }(37,5 \%), \text { felandral } \\
(26,0 \%), \tau \text {-terpineno }(6,6 \%) \text { e } \\
\beta \text {-cimeno }(5,2 \%)\end{array}$ & C. albicans & Mamadalieva et al., 2019 \\
\hline Thymus daenensis & $\begin{array}{l}\text { timol }(73,86 \%) \text { e carvacrol } \\
\qquad(51,89 \%)\end{array}$ & $\begin{array}{l}\text { C. albicans }(M I C=0,17 \\
\text { a } 0,58 \mathrm{mg} / \mathrm{ml})\end{array}$ & Elahian et al., 2021 \\
\hline Thymus leptobotrys & $\begin{array}{c}\text { carvacrol } \\
(73,68 \%), \text { p-cimeno }(8,68 \%) \text { e } \\
\gamma \text {-terpineno }(4,14 \%)\end{array}$ & $\begin{array}{l}\text { C. albicans } e \\
\text { C. tropicalis }\end{array}$ & Oubihi et al., 2020 \\
\hline Thymus linearis & $\begin{array}{l}\text { timol }(54,9 \%), \gamma \text {-terpineno } \\
(16,6 \%) \text { e p-cimeno }(5,2 \%)\end{array}$ & $\begin{array}{l}\text { C. albicans }(M I C=990 \\
\mu g / m L) \\
\text { C. glabrata }(M I C=990 \\
\mu g / m L) \\
\text { e C. tropicalis }(M I C= \\
240 \mu g / m L)\end{array}$ & Kumar et al., 2020 \\
\hline $\begin{array}{l}\text { Thymus munbyanus subsp. } \\
\text { coloratus }\end{array}$ & $\begin{array}{l}\text { borneol ( } 44,8 \% \text { e } 31,2 \% \text { de } \\
\text { flores e partes aéreas, } \\
\text { respectivamente, cânfora }(5,7 \% \\
\text { e } 13,6 \%, \text { respectivamente), } \\
\text { canfeno (3,6\% e 7,5\%, } \\
\text { respectivamente), }\end{array}$ & C. albicans & Bendif et al., 2017 \\
\hline Thymus pannonicus & $\begin{array}{l}\text { citral, 3-octanona, 1-octen-3-ol, } \\
\text { linalol e 1,8-cineol }\end{array}$ & $\begin{array}{l}\text { C. albicans } \\
\text { (MIC 31,25-62,50 L / } \\
\mathrm{mL})\end{array}$ & Arsenijevic et al., 2016 \\
\hline Thymus pulegioides & $\begin{array}{c}\text { a-Citral }(27,10 \%) \text { e bcitral } \\
(17,11 \%)\end{array}$ & C. albicans & Shanaida et al., 2021 \\
\hline Thymus serpyllum & $\begin{array}{c}\text { Timol }(52,56 \%) \text { e Éter metílico } \\
\text { de isotimol }(11,28 \%)\end{array}$ & C. albicans & Shanaida et al., 2021 \\
\hline Thymus syriacus & Carvacrol $(57,3 \%)$ & $\begin{array}{l}\text { C. albicans }(M I C=0,8 \\
m g / m L)\end{array}$ & Hafi et al., 2017 \\
\hline Thymus vulgaris & $\begin{array}{c}\text { timol }(75,2 \%) \\
\text { carvacrol }(7,7 \%) \text { e p-cimeno } \\
(6,3 \%)\end{array}$ & & \\
\hline
\end{tabular}




\begin{tabular}{|c|c|c|c|}
\hline Thymus vulgaris & $\begin{array}{l}\text { carvacrol }(56,8 \%), \text { seguido por } \\
\text { p-cimeno }(12,8 \%), \gamma \text {-terpineno } \\
\quad(11,17 \%) \text { e timol }(3,99 \%)\end{array}$ & $\begin{array}{l}\text { C. albicans e } C \text {. } \\
\text { parapsilosis }(\text { MIC - } 0,3 \text { a } \\
0,15 \mu \mathrm{L} / \mathrm{mL})\end{array}$ & Boukhatem et al., 2020 \\
\hline Thymus vulgaris & $\begin{array}{l}\text { timol }(52,6 \%), \mathrm{p} \text {-cimeno } \\
(15,3 \%)\end{array}$ & C. albicans e C. famata & Ebani et al., 2018 \\
\hline Thymus vulgaris & $\begin{array}{c}\rho \text {-cimeno }(40,0 \pm 3 \%) \text { timol } \\
(32,0 \pm 2 \%)\end{array}$ & C. albicans & Proškovcová et al., 2021 \\
\hline Thymus vulgaris & $\begin{array}{c}\text { Timol }(47,33 \%) \text {, o-Cimeno } \\
(14,34 \%) \text { e } \gamma \text {-terpineno } \\
(11,57 \%)\end{array}$ & C. albicans & Shanaida et al., 2021 \\
\hline Vitex agnus-castus & Eucaliptol $(20,5 \%)$ & $\begin{array}{l}\text { C. albicans } \\
(M I C=512 \mu \mathrm{g} / \mathrm{mL})\end{array}$ & Khoury et al., 2016 \\
\hline Zataria multiflora & $\begin{array}{c}\text { timol }(52,8 \%) \text { seguido por } \\
\text { cimeno (o-) } \\
(13,89 \%) \text { e carvacrol }(5,96 \%) \text {. }\end{array}$ & C. albicans & Ardekani et al., 2019 \\
\hline Zataria multiflora & $\begin{array}{l}\text { timol }(34,41-54,35 \%), \mathrm{p}- \\
\text { cimeno }(9,49-19,85 \%), \gamma- \\
\text { terpineno }(7,34-16,70 \%) \\
\text { carvacrol }(5,35-15,34 \%) \\
\text { e } \alpha \text {-pineno }(1,63-5,25 \%)\end{array}$ & C. albicans & Niczad et al., 2019 \\
\hline Ziziphora tenuior & $\begin{array}{l}\text { pulegone }(46,8 \%) \text {, p-menth-3- } \\
\text { en-8-ol } \\
(12,5 \%) \text {, isomentona }(6,6 \%) \text { e } \\
\text { 8-hidroximentona }(6,2 \%)\end{array}$ & $\begin{array}{l}\text { C. albicans, } C \text {. tropicalis } \\
\text { e } C \text {. parapsilosis }(\text { MIC }= \\
1.25 \mu \mathrm{l} / \mathrm{mL})\end{array}$ & Abu-Darwish et al., 2016 \\
\hline
\end{tabular}

Fonte: Autores (2021).

\section{Conclusão}

A revisão discute dados obtidos de 56 artigos sobre a atividade anti-Candida de óleos essências de 71 de plantas de Lamiaceae. Os óleos essenciais das espécies mostraram boa atividade anti-Candida (CIMs <1000 $\mu \mathrm{g} / \mathrm{mL}$ ). A triagem de espécies vegetais com atividade anti-Candida pode representar um importante direcionamento para uma abordagem taxonômica visando rastrear espécies relacionadas que podem conter compostos químicos importantes em seus óleos essências, método que já se mostrou muito bem sucedido. Os óleos essenciais podem ser de grande importância no tratamento de fungos multirresistentes a medicamentos no futuro.

\section{Considerações Finais}

Diante do exposto, é imprescindível que as pesquisas que envolvam a atividade química e biológica de óleos essenciais sejam melhor investigadas para que novos fármacos possam ser descobertos possibilitando a obtenção de produtos menos agressivos, de menor custo e, portanto, mais acessíveis as populações. 


\section{Referências}

Abu-Darwish, M. S., Cabral, C., Goncalves, M. J., Cavaleiro, C., Cruz, M. T., Paoli, M., ... \& Salgueiro, L. (2016). Ziziphora tenuior L. essential oil from dana biosphere reserve (Southern Jordan); chemical characterization and assessment of biological activities. Journal of ethnopharmacology, 194, 963-970.

Al Hafi, M., El Beyrouthy, M., Ouaini, N., Stien, D., Rutledge, D., \& Chaillou, S. (2017). Chemical composition and antimicrobial activity of Satureja, Thymus, and Thymbra species grown in Lebanon. Chemistry \& biodiversity, 14(5), e1600236.

Albayrak, S., \& Aksoy, A. (2019). Phenolic contents and biological activity of endemic Origanum minutiflorum grown in Turkey. Indian J. Pharm. Edu. Res., 53(1), 160-170.

Alizadeh, A. (2016). Essential oil constituents and biological activities of different ecotypes of Satureja bachtiarica Bunge. as a traditional herbal drug in Southwestern Iran. Journal of Essential Oil Bearing Plants, 19(6), 1328-1339.

Andrys, D., Kulpa, D., Grzeszczuk, M., \& Białecka, B. (2018). Influence of jasmonic acid on the growth and antimicrobial and antioxidant activities of Lavandula angustifolia Mill. propagated in vitro. Folia Horticulturae, 30(1), 3-13.

Arantes, S., Candeias, F., Lopes, O., Lima, M., Pereira, M., Tinoco, T., ... \& Martins, M. R. (2016). Pharmacological and toxicological studies of essential oil of Lavandula stoechas subsp. luisieri. Planta medica, 82(14), 1266-1273.

Ardekani, N. T., Khorram, M., Zomorodian, K., Yazdanpanah, S., Veisi, H., \& Veisi, H. (2019). Evaluation of electrospun poly (vinyl alcohol)-based nanofiber mats incorporated with Zataria multiflora essential oil as potential wound dressing. International journal of biological macromolecules, $125,743-750$.

Armstrong-James, D., Brown, G. D., Netea, M. G., Zelante, T., Gresnigt, M. S., van de Veerdonk, F. L., \& Levitz, S. M. (2017). Immunotherapeutic approaches to treatment of fungal diseases. The Lancet Infectious Diseases, 17(12), e393-e402.

Arsenijević, J., Drobac, M., Šoštarić, I., Ražić, S., Milenković, M., Couladis, M., \& Maksimović, Z. (2016). Bioactivity of herbal tea of Hungarian thyme based on the composition of volatiles and polyphenolics. Industrial Crops and Products, 89, 14-20.

Baj, T., Biernasiuk, A., Wróbel, R., \& Malm, A. (2020). Chemical composition and in vitro activity of Origanum vulgare L., Satureja hortensis L., Thymus serpyllum L. and Thymus vulgaris L. essential oils towards oral isolates of Candida albicans and Candida glabrata. Open Chemistry, 18(1), 108-118.

Benali, T., Habbadi, K., Bouyahya, A., Khabbach, A., Marmouzi, I., Aanniz, T., ... \& Hammani, K. (2021). Phytochemical Analysis and Study of Antioxidant, Anticandidal, and Antibacterial Activities of Teucrium polium subsp. polium and Micromeria graeca (Lamiaceae) Essential Oils from Northern Morocco. Evidence-Based Complementary and Alternative Medicine, 2021.

Bendif, H., Boudjeniba, M., Miara, M. D., Biqiku, L., Bramucci, M., Lupidi, G., ... \& Maggi, F. (2017). Essential Oil of Thymus munbyanus subsp. coloratus from Algeria: Chemotypification and in vitro Biological Activities. Chemistry \& biodiversity, 14(3), e1600299.

Bezerra, J. W. A., Rodrigues, F. C., Costa, A. R., Pereira, K. S., Vieira, N. R., de Oliveira Lôbo, G., ... \& Braga, M. F. B. M. (2020). Mesosphaerum suaveolens (Lamiacae): Source of antimicrobial and antioxidant compounds. Research, Society and Development, 9(8), e575986161-e575986161.

Bogavac, M. A., Karaman, M. A., Suđi, J. J., Radovanović, B. B., Janjušević, L. N., Ćetković, N. B., \& Tešanović, K. D. (2017). Antimicrobial potential of Rosmarinus officinalis commercial essential oil in the treatment of vaginal infections in pregnant women. Natural product communications, $12(1)$, 1934578 X1701200136.

Bona, E., Cantamessa, S., Pavan, M., Novello, G., Massa, N., Rocchetti, A., ... \& Gamalero, E. (2016). Sensitivity of Candida albicans to essential oils: are they an alternative to antifungal agents?. Journal of applied microbiology, 121(6), 1530-1545.

Boukhatem, M. N., Darwish, N. H., Sudha, T., Bahlouli, S., Kellou, D., Benelmouffok, A. B., ... \& Mousa, S. A. (2020). In vitro antifungal and topical antiinflammatory properties of essential oil from wild-growing thymus vulgaris (Lamiaceae) used for medicinal purposes in algeria: A new source of carvacrol. Scientia Pharmaceutica, 88(3), 33.

Božović, M., Garzoli, S., Baldisserotto, A., Romagnoli, C., Pepi, F., Cesa, S., ... \& Ragno, R. (2018). Melissa officinalis L. subsp. altissima (Sibth. \& Sm.) Arcang. essential oil: Chemical composition and preliminary antimicrobial investigation of samples obtained at different harvesting periods and by fractionated extractions. Industrial Crops and Products, 117, 317-321.

Casiglia, S., Bruno, M., Fontana, G., \& Senatore, F. (2017). Chemical Composition of the Essential Oil of Mentha pulegium Growing Wild in Sicily and its Activity on Microorganisms Affecting Historical Art Crafts. Natural Product Communications, 12(8), 1934578 X1701200840.

Chaib, F., Allali, H., Bennaceur, M., \& Flamini, G. (2017). Chemical composition and antimicrobial activity of essential oils from the aerial parts of Asteriscus graveolens (Forssk.) Less. and Pulicaria incisa (Lam.) DC.: Two Asteraceae herbs growing wild in the Hoggar. Chemistry \& biodiversity, 14(8), e1700092.

Çolak, N. U., Yıldırım, S., Bozdeveci, A., Yaylı, N., Çoşkunçelebi, K., Fandaklı, S., \& Yaşar, A. (2018). Essential oil composition, antimicrobial and antioxidant activities of Salvia staminea.

Cutillas, A. B., Carrasco, A., Martinez-Gutierrez, R., Tomas, V., \& Tudela, J. (2018). Rosmarinus officinalis L. essential oils from Spain: Composition, antioxidant capacity, lipoxygenase and acetylcholinesterase inhibitory capacities, and antimicrobial activities. Plant Biosystems-An International Journal Dealing with all Aspects of Plant Biology, 152(6), 1282-1292.

Debbabi, H., El Mokni, R., Chaieb, I., Nardoni, S., Maggi, F., Caprioli, G., \& Hammami, S. (2020). Chemical Composition, Antifungal and Insecticidal Activities of the Essential Oils from Tunisian Clinopodium nepeta subsp. nepeta and Clinopodium nepeta subsp. glandulosum. Molecules, $25(9), 2137$.

Dorsaz, S., Snäkä, T., Favre-Godal, Q., Maudens, P., Boulens, N., Furrer, P., ... \& Sanglard, D. (2017). Identification and mode of action of a plant natural product targeting human fungal pathogens. Antimicrobial agents and chemotherapy, 61(9), e00829-17. 
Duarte, A. E., \& de Menezes, I. R. (2016). Bezerra Morais Braga MF et al. Antimicrobial Activity and Modulatory Effect of Essential Oil from the Leaf of Rhaphiodon echinus (Nees \&amp; Mart) Schauer on Some Antimicrobial Drugs. Molecules, 21(6).

Ebani, V. V., Nardoni, S., Bertelloni, F., Pistelli, L., \& Mancianti, F. (2018). Antimicrobial activity of five essential oils against bacteria and fungi responsible for urinary tract infections. Molecules, 23(7), 1668.

Elahian, F., Garshasbi, M., Mehri Asiabar, Z., Gholamian Dehkordi, N., Yazdinezhad, A., \& Mirzaei, S. A. (2021). Ecotypic Variations Affected the Biological Effectiveness of Thymus daenensis Celak Essential Oil. Evidence-Based Complementary and Alternative Medicine, 2021.

Ferrante, C., Recinella, L., Ronci, M., Orlando, G., Di Simone, S., Brunetti, L., ... \& Menghini, L. (2019). Protective effects induced by alcoholic Phlomis fruticosa and Phlomis herba-venti extracts in isolated rat colon: Focus on antioxidant, anti-inflammatory, and antimicrobial activities in vitro. Phytotherapy Research, 33(9), 2387-2400.

Fikry, S., Khalil, N., \& Salama, O. (2019). Chemical profiling, biostatic and biocidal dynamics of Origanum vulgare L. essential oil. AMB Express, 9(1), 1-10.

Ghavam, M., Manconi, M., Manca, M. L., \& Bacchetta, G. (2021). Extraction of essential oil from Dracocephalum kotschyi Boiss. (Lamiaceae), identification of two active compounds and evaluation of the antimicrobial properties. Journal of Ethnopharmacology, 267, 113513.

Günbatan, T., Demirci, B., Gürbüz, İ., Demircib, F., \& Özkanc, A. M. G. (2017). Comparison of Volatiles of Sideritis caesarea Specimens Collected from Different Localities in Turkey. Natural Product Communications, 12(10), 1934578X1701201029.

Guo, J., Zhang, M., Qiao, D., Shen, H., Wang, L., Wang, D., ... \& Wu, W. (2021). Prevalence and Antifungal Susceptibility of Candida parapsilosis Species Complex in Eastern China: A 15-Year Retrospective Study by ECIFIG. Frontiers in microbiology, 12, 249.

Iseppi, R., Tardugno, R., Brighenti, V., Benvenuti, S., Sabia, C., Pellati, F., \& Messi, P. (2020). Phytochemical Composition and In Vitro Antimicrobial Activity of Essential Oils from the Lamiaceae Family against Streptococcus agalactiae and Candida albicans Biofilms. Antibiotics, 9(9), 592.

Jafari, F., Farmani, F., Zomorodian, K., Moein, M., Faridi, P., \& Zarshenas, M. M. (2018). A study on essential oil chemical compositions, antioxidant, and antimicrobial activities of native and endemic Satureja Species Growing in Iran. Pharmaceutical Chemistry Journal, 52(1), 63-68.

Justus, B., Almeida, V. P. D., Gonçalves, M. M., Assunção, D. P. D. S. F. D., Borsato, D. M., Arana, A. F. M., ... \& Farago, P. V. (2018). Chemical composition and biological activities of the essential oil and anatomical markers of Lavandula dentata L. cultivated in Brazil. Brazilian Archives of Biology and Technology, 61.

Karadağ, A. E., Demirci, B., Kültür, Ş., Demirci, F., \& Başer, K. H. C. (2020). Antimicrobial, anticholinesterase evaluation and chemical characterization of essential oil Phlomis kurdica Rech. fil. Growing in Turkey. Journal of Essential Oil Research, 32(3), 242-246.

Karpiński, T. M. (2020). Essential oils of Lamiaceae family plants as antifungals. Biomolecules, 10(1), 103.

Khoury, M., Stien, D., Eparvier, V., Ouaini, N., \& El Beyrouthy, M. (2016). Report on the medicinal use of eleven Lamiaceae species in Lebanon and rationalization of their antimicrobial potential by examination of the chemical composition and antimicrobial activity of their essential oils. Evidence-Based Complementary and Alternative Medicine, 2016.

Kiashi, F., Hadjiakhoondi, A., Tofighi, Z., Khanavi, M., Ajani, Y., Ahmadi Koulaei, S., \& Yassa*, N. (2021). Compositions of essential oils and some biological properties of Stachys laxa Boiss. \& Buhse and S. byzantina K. Koch. Research Journal of Pharmacognosy, 8(2), 5-15.

Kirmizibekmez, H., Karaca, N., Demirci, B., \& Demirci, F. (2017). Characterization of Sideritis trojana Bornm. essential oil and its antimicrobial activity. Marmara Pharmaceutical Journal, 21(4), 860-865.

Kumar, A., Kamal, A., Singh, S., Padalia, R. C., Tandon, S., Chauhan, A., ... \& Verma, R. S. (2020). Chemical composition, antimicrobial activity, kinetics and mechanism of action of Himalayan-thyme (Thymus linearis Benth.). Journal of Essential Oil Research, 32(1), 59-68.

Liu, S., Hou, Y., Chen, X., Gao, Y., Li, H., \& Sun, S. (2014). Combination of fluconazole with non-antifungal agents: a promising approach to cope with resistant Candida albicans infections and insight into new antifungal agent discovery. International journal of antimicrobial agents, 43(5), 395-402.

Lorenzo-Leal, A. C., Palou, E., López-Malo, A., \& Bach, H. (2019). Antimicrobial, cytotoxic, and anti-inflammatory activities of Pimenta dioica and Rosmarinus officinalis essential oils. BioMed research international, 2019.

MALINOVSKÁ, Zuzana. Antibiofilm activity of selected plant essential oils from the Lamiaceae family against Candida albicans clinical isolates. Annals of Agricultural and Environmental Medicine, v. 28, n. 2, p. 260-266, 2021.

Mamadalieva, N. Z., Youssef, F. S., Ashour, M. L., Sasmakov, S. A., Tiezzi, A., \& Azimova, S. S. (2019). Chemical composition, antimicrobial and antioxidant activities of the essential oils of three Uzbek Lamiaceae species. Natural product research, 33(16), 2394-2397.

Marak, M. B., \& Dhanashree, B. (2018). Antifungal susceptibility and biofilm production of Candida spp. isolated from clinical samples. International journal of microbiology, 2018.

Marino, A., Nostro, A., Mandras, N., Roana, J., Ginestra, G., Miceli, N., ... \& Tullio, V. (2020). Evaluation of antimicrobial activity of the hydrolate of Coridothymus capitatus (L.) Reichenb. fil.(Lamiaceae) alone and in combination with antimicrobial agents. BMC complementary medicine and therapies, 20(1), $1-11$.

Mback, M. N., Agnaniet, H., Nguimatsia, F., Dongmo, P. M. J., Fokou, J. B. H., Bakarnga-Via, I., ... \& Menut, C. (2016). Optimization of antifungal activity of Aeollanthus heliotropioides oliv essential oil and Time Kill Kinetic Assay. Journal de mycologie medicale, 26(3), 233-243.

McCarty, T. P., \& Pappas, P. G. (2016). Invasive candidiasis. Infectious Disease Clinics, 30(1), 103-124. 
Medici, N. P., \& Del Poeta, M. (2015). New insights on the development of fungal vaccines: from immunity to recent challenges. Memorias do Instituto Oswaldo Cruz, 110, 966-973.

Milenković, M., Stošović, J., \& Slavkovska, V. (2018). Synergy between essential oils of Calamintha species (Lamiaceae) and antibiotics. Natural Product Communications, 13(3), 1934578X1801300325.

Monzote, L., Scherbakov, A. M., Scull, R., Gutiérrez, Y. I., Satyal, P., Cos, P., ... \& Setzer, W. N. (2020). Pharmacological assessment of the carvacrol chemotype essential oil from Plectranthus amboinicus growing in Cuba. Natural Product Communications, 15(10), 1934578X20962233.

Müller-Sepúlveda, A., Chevecich, C. C., Jara, J. A., Belmar, C., Sandoval, P., Meyer, R. S., ... \& Molina-Berríos, A. (2020). Chemical Characterization of Lavandula dentata Essential Oil Cultivated in Chile and Its Antibiofilm Effect against Candida albicans. Planta Medica, 86(16), 1225-1234.

Najibzadeh, T., \& Yadegary, M. H. (2018). Antifungal effect of Satureja khuzestanica Jamzad essential oil on oral candidiasis in immunosuppressed rats. Herba Polonica, 64(3).

Nami, S., Mohammadi, R., Vakili, M., Khezripour, K., Mirzaei, H., \& Morovati, H. (2019). Fungal vaccines, mechanism of actions and immunology: a comprehensive review. Biomedicine \& Pharmacotherapy, 109, 333-344.

Niczad, A., Sharafzadeh, S., Alizadeh, A., Amiri, B., \& Bazrafshan, F. (2019). Variability in essential oil constituent, phenolic content, antioxidant and antimicrobial activities of different ecotypes of Zataria multiflora Boiss. from Iran. Journal of Essential Oil Bearing Plants, 22(6), 1435-1449.

Oubihi, A., Ouryemchi, I., Nounah, I., Tarfaoui, K., Harhar, H., Ouhssine, M., \& Guessous, Z. (2020). Chemical composition, antibacterial and antifungal activities of Thymus leptobotrys Murb essential oil. Advances in Traditional Medicine, 20(4), 673-679.

Gupta, S., \& Goyal, R. K. (2017). Species Distribution and Antifungal Drug Susceptibility of Candida in Clinical Isolates from a Tertiary Care Centre at Bareilly. IOSR-JDMS, 1(2), 57-61.

Piras, A., Gonçalves, M. J., Alves, J., Falconieri, D., Porcedda, S., Maxia, A., \& Salgueiro, L. (2018). Ocimum tenuiflorum L. and Ocimum basilicum L., two spices of Lamiaceae family with bioactive essential oils. Industrial Crops and Products, 113, 89-97.

Potente, G., Bonvicini, F., Gentilomi, G. A., \& Antognoni, F. (2020). Anti-Candida Activity of Essential Oils from Lamiaceae Plants from the Mediterranean Area and the Middle East. Antibiotics, 9(7), 395.

Ramírez, J., Gilardoni, G., Jácome, M., Montesinos, J., Rodolfi, M., Guglielminetti, M. L., ... \& Vidari, G. (2017). Chemical composition, enantiomeric analysis, AEDA sensorial evaluation and antifungal activity of the essential oil from the Ecuadorian plant Lepechinia mutica Benth (Lamiaceae). Chemistry \& biodiversity, 14(12), e1700292.

Sabbobeh, R., Hejaz, H., Jahajha, A., Al-Akhras, S., Al-Jaas, H., \& Abu-Lafi, S. (2016). Antioxidant an antimicrobial activities of the leaf extract of Salvia palaestina. J. Appl. Pharm. Sci, 6, 76-82.

Salameh, N., Shraim, N., Jaradat, N., El Masri, M., Adwan, L., K'aibni, S., ... \& AbuAlhasan, M. (2020). Screening of antioxidant and antimicrobial activity of Micromeria fruticosa serpyllifolia volatile oils: A comparative study of plants collected from different regions of west Bank, Palestine. BioMed research international, 2020

Salehi, B., Mishra, A. P., Shukla, I., Sharifi-Rad, M., Contreras, M. D. M., Segura-Carretero, A., ... \& Sharifi-Rad, J. (2018). Thymol, thyme, and other plant sources: Health and potential uses. Phytotherapy Research, 32(9), 1688-1706.

Shakeri, A., Khakdan, F., Soheili, V., Sahebkar, A., Shaddel, R., \& Asili, J. (2016). Volatile composition, antimicrobial, cytotoxic and antioxidant evaluation of the essential oil from Nepeta sintenisii Bornm. Industrial Crops and Products, 84, 224-229.

Shanaida, M., Hudz, N., Białoń, M., Kryvtsowa, M., Svydenko, L., Filipska, A., \& Wieczorek, P. P. (2021). Chromatographic profiles and antimicrobial activity of the essential oils obtained from some species and cultivars of the Mentheae tribe (Lamiaceae). Saudi journal of biological sciences, 28(11), 6145-6152.

Sharma, A., Cooper, R., Bhardwaj, G., \& Cannoo, D. S. (2021). The genus Nepeta: Traditional uses, phytochemicals and pharmacological properties. Journal of Ethnopharmacology, 268, 113679.

Spampinato, C., \& Leonardi, D. (2013). Candida infections, causes, targets, and resistance mechanisms: traditional and alternative antifungal agents. BioMed research international, 2013.

Stanojevic, L. P., Marjanovic-Balaban, Z. R., Kalaba, V. D., Stanojevic, J. S., Cvetkovic, D. J., \& Cakic, M. D. (2017). Chemical composition, antioxidant and antimicrobial activity of basil (Ocimum basilicum L.) essential oil. Journal of Essential Oil Bearing Plants, 20(6), 1557-1569.

Tadić, V., Oliva, A., Božović, M., Cipolla, A., De Angelis, M., Vullo, V., ... \& Ragno, R. (2017). Chemical and antimicrobial analyses of Sideritis romana L. subsp. purpurea (Tal. ex Benth.) Heywood, an endemic of the Western Balkan. Molecules, 22(9), 1395.

Tsay, S. V., Mu, Y., Williams, S., Epson, E., Nadle, J., Bamberg, W. M., ... \& Vallabhaneni, S. (2020). Burden of candidemia in the united states, 2017. Clinical Infectious Diseases, 71(9), e449-e453.

Yiğit Hanoğlu, D., Hanoğlu, A., Güvenir, M., Süer, K., Demirci, B., Başer, K. H. C., \& Özkum Yavuz, D. (2017). Chemical composition and antimicrobial activity of the essential oil of Sideritis cypria Post endemic in Northern Cyprus. Journal of Essential Oil Research, 29(3), 228-232.

Zhou, S., Wei, C., Zhang, C., Han, C., Kuchkarova, N., \& Shao, H. (2019). Chemical composition, phytotoxic, antimicrobial and insecticidal activity of the essential oils of Dracocephalum integrifolium. Toxins, 11(10), 598

Zuza-Alves, D. L., Silva-Rocha, W. P., \& Chaves, G. M. (2017). An update on Candida tropicalis based on basic and clinical approaches. Frontiers in microbiology, 8, 1927. 